\title{
Tendencias recientes de las principales causas de muerte en Navarra. 1995-2004
}

\author{
Recent trends of the main causes of death in Navarre. 1995-2004
}

\author{
C. Moreno-Iribas, Y. Floristán, N. Egüés
}

\section{RESUMEN}

Se presentan las estadísticas de mortalidad de Navarra para el periodo 1995-2004 por sexo, edad y causa de muerte.

Material y métodos. Los datos proceden de los boletines estadísticos de defunción. Se comparan el periodo 1995-1999 con el periodo 2000-2004, utilizando las tasas ajustadas a la población de Navarra del año 2001 y la tasa de años potenciales de vida perdidos entre 0-70 años ajustada a la población europea.

Resultados. La esperanza de vida al nacer en el periodo 2000-2004 fue de 77,9 años en los hombres y 85 años en las mujeres, esta última una de las más altas registradas a nivel mundial. El riesgo de muerte ha diminuido en prácticamente todos los grupos de edad, destacando un descenso de la mortalidad del $40 \%$ en el grupo de 25-34 años a costa del sida. La mortalidad infantil bajó hasta 3,3 por 1.000 recién nacidos vivos. Ha habido un importante incremento de la mortalidad por cáncer de pulmón entre las mujeres menores de 65 años (46\%) en el quinquenio 2000-04 respecto al anterior y un fuerte descenso de la mortalidad por cáncer de mama $(45,4 \%)$. Han continuado disminuyendo en ambos sexos la mortalidad por enfermedades cerebrovasculares, enfermedad isquémica del corazón, insuficiencia cardiaca y arterioesclerosis así como la mortalidad por úlceras y cirrosis. Las tasas de mortalidad de Navarra de todos los grandes grupos de causas son más bajas que las de España excepto la tasa de mortalidad por causas externas.

Conclusiones. El incremento de la mortalidad por cáncer de pulmón y de otros cánceres relacionados con el tabaquismo debido a la extensión del consumo en las últimas décadas puede influir negativamente en la esperanza de vida de las mujeres de Navarra en los próximos años. La prevención de la mortalidad por causas externas y la prevención de las muertes ligadas al consumo de tabaco son dos prioridades que se desprenden de los datos de mortalidad de esta comunidad autónoma.

Palabras clave. Mortalidad. Años potenciales de vida perdidos. Mortalidad infantil. Esperanza de vida al nacer.

\begin{abstract}
We present the statistics for mortality in Navarre for the 1995-2004 period by sex, age and cause of death.

Methods. The data proceeds from the statistical bulletins on death. The 1995-1999 period is compared with the 2000-2004 period, using adjusted rates to the population of Navarre for the year 2001 and the rate of potential years of life lost between 0 70 years adjusted to the European population.
\end{abstract}

Results. Life expectancy at birth in the 2000-2004 period was 77.9 years in men and 85 years in women, the latter being one of the highest registered at the world level. Infant mortality fell to 3.3 per 1,000 living newborn infants. The risk of death has fallen in practically all age groups, with a notable fall in mortality of $40 \%$ in the 25-34 group at the cost of AIDS. There has been a significant increase of mortality due to lung cancer amongst women under 65 years of age (46\%) in the 2000-2004 period with respect to the previous five year period and a sharp fall in mortality due to breast cancer (45.4\%). In both sexes there has been a continued fall of mortality due to cerebrovascular diseases, ischaemic heart diseases, cardiac failure and arteriosclerosis and of mortality due to ulcer and cirrhosis. The rates of mortality in Navarre in all the main groups of causes are lower than those of Europe, except for the rate of mortality due to external causes.

Conclusions. The increase of mortality due to lung cancer and other cancers related to smoking, due to the spread of consumption in recent decades, could have a negative influence on the life expectancy of women in Navarre in coming years. The prevention of mortality due to external causes and the prevention of deaths linked to tobacco consumption are the two priorities that emerge from the data on mortality in this autonomous community.

Key words. Mortality. Potential years of life lost. Infant mortality. Life expectancy at birth.

An. Sist. Sanit. Navar. 2006; 29 (3): 399-414.

Instituto de Salud Pública de Navarra. Pamplona

Aceptado para su publicación el 9 de junio de 2006.

\section{Correspondencia:}

Conchi Moreno Iribas

Instituto de Salud Pública

c/ Leyre 15

31003 Pamplona

Tfno: 848423470

E-mail: mmorenoi@cfnavarra.es 


\section{INTRODUCCIÓN}

El estudio de la mortalidad es un componente necesario del análisis y vigilancia de la situación de salud y punto de referencia obligado en la evaluación de las políticas sanitarias. Se presentan los indicadores de mortalidad para Navarra en el periodo 1995-2004 que describen la magnitud y la evolución de las principales causas de muerte y que resume la información fundamental del patrón de mortalidad de Navarra. Se realizan algunas comparaciones con datos de España y la Unión Europea (UE).

\section{MATERIAL Y MÉTODOS}

El origen de los datos son los boletines estadísticos de defunción de los residentes en Navarra fallecidos en Navarra o en otras Comunidades Autónomas. La codificación de la causa básica de defunción se ha realizado utilizando la Clasificación Internacional de Enfermedades (CIE), la novena revisión entre 1995-1998 y la décima revisión a partir de 1999, entendiendo como causa básica de defunción: "todas aquellas enfermedades, estados morbosos o lesiones que produjeron la muerte o que contribuyeron a ella, o las circunstancias del accidente o violencia que produjo la lesión fatal". El Instituto Nacional de Estadística en colaboración con los registros de mortalidad autonómicos adoptó determinados criterios complementarios de equivalencia entre la novena y décima revisión de la CIE para elaborar una lista reducida de 102 causas de muerte, que se ha utilizado en este trabajo. Como denominadores se han utilizado las poblaciones estimadas por el Instituto de Estadística de Navarra ${ }^{1}$.

Se ha calculado la esperanza de vida como la media de años que una persona de determinada edad puede esperar vivir asumiendo que las tasas específicas de mortalidad permanezcan constantes (es decir, que se mantengan en las tasas observadas en el periodo de estudio) para dar mayor estabilidad a la estimación. Se ha calculado la esperanza de vida para cada uno de los sexos y quinquenios estudiados (1995-1999 y 2000-2004) presentándose los datos referidos a la esperanza de vida al nacer.

Para mejorar la comparabilidad de los datos entre los dos periodos estudiados con otros países y, puesto que el objetivo es medir las tasas de mortalidad independientemente de la estructura de las poblaciones, se han calculado las tasas ajustadas a la población de Navarra en el año 2001 y las tasas ajustadas a la población estándar europea. Para analizar la mortalidad prematura se ha calculado la tasa de años potenciales de vida perdidos entre 0 70 años ajustada a la población europea (APVP). Las comparaciones con datos de España y Europa se han realizado utilizando los datos publicados por el Instituto Nacional de Estadística ${ }^{2}$ y Eurostat ${ }^{3-5}$.

El análisis estadístico se ha realizado con el paquete estadístico SPSS/PC+.

\section{MORTALIDAD GENERAL Y ESPERANZA DE VIDA}

En el año 2004, último año estudiado, fallecieron en Navarra 2.598 hombres y 2.401 mujeres. Entre los hombres, el 19,4\% de los muertos tenían menos de 65 años y el 24,5\% más de 85 años, mientras que entre las mujeres estos porcentajes fueron de $10 \%$ y $50 \%$, respectivamente. La figura 1 recoge la evolución de las tasas ajustadas de mortalidad desde 1995 hasta 2004 en Navarra y en España. A lo largo de todo el periodo las tasas de mortalidad descendieron en ambos sexos y además hay que señalar que las tasas de Navarra en ambos sexos son más bajas que las de España.

En el último quinquenio, 2000-2004, la esperanza de vida al nacer en Navarra fue de 77,9 años en los hombres y 85 años en las mujeres, por encima de la observada en el conjunto de la UE- $15^{3}$ donde, según Eurostat, la esperanza de vida al nacer en el año 2002 fue de 75,9 años en los hombres y 81,7 años en las mujeres y la de España de 76,2 y 82,9 años respectivamente.

A nivel mundial y para el año 2002, los países con más alta esperanza de vida al nacer entre los hombres fueron Islandia 78,7 , Suecia 77,7 y Japón 77,5 , según publica Eurostat ${ }^{3}$. Entre las mujeres destacan las japonesas con una esperanza de vida al nacer de 84,3 años y en Europa las mujeres de Francia con 83,0 años y España, 82,9 años. 


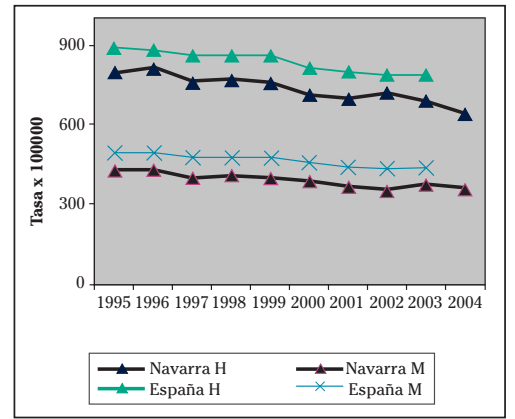

\begin{tabular}{ccccc}
\hline Año & Navarra $\mathbf{H}$ & Navarra M & España $\mathbf{H}$ & España $\mathbf{M}$ \\
\hline $\mathbf{1 9 9 5}$ & 797,1 & 428,2 & 888,5 & 495,1 \\
$\mathbf{1 9 9 6}$ & 805,2 & 425,5 & 884,3 & 489,5 \\
$\mathbf{1 9 9 7}$ & 758,1 & 400,6 & 857,8 & 475,8 \\
$\mathbf{1 9 9 8}$ & 761,9 & 401,2 & 863,6 & 477,7 \\
$\mathbf{1 9 9 9}$ & 755,1 & 400,3 & 861,6 & 477,6 \\
$\mathbf{2 0 0 0}$ & 715,5 & 388,7 & 819,7 & 454,5 \\
$\mathbf{2 0 0 1}$ & 697,3 & 365,8 & 803,3 & 442,0 \\
$\mathbf{2 0 0 2}$ & 718,6 & 352,0 & 787,7 & 436,2 \\
$\mathbf{2 0 0 3}$ & 690,9 & 376,9 & 784,6 & 442,9 \\
$\mathbf{2 0 0 4}$ & 640,7 & 358,4 & nd & nd \\
\hline
\end{tabular}

Figura 1. Mortalidad por todas las causas en Navarra y España en el periodo 1995-2004. Tasas ajustadas por edad a la población europea por 100.000: Hombres (H) y Mujeres (M).

La esperanza de vida de las mujeres de Navarra es de 7,1 años más alta que la de los hombres. Esta gran diferencia entre la esperanza de vida de los hombres y mujeres ha sido descrita también en Francia donde la diferencia es de 7,6 años. En el otro extremo se encuentran países como Suecia donde la diferencia entre sexos es de sólo 5,1 años ${ }^{3}$.

\section{MORTALIDAD INFANTIL}

La mortalidad infantil ha sido durante muchos años reconocida como un importante indicador de salud pública y se refiere al riesgo de muerte en el primer año de vida. Los datos de la tabla 1 muestran las tasas de mortalidad infantil en el periodo 1995-2004 en Navarra; la tasa descendió un $28,8 \%$ entre $1995-1999$ y 2000-2004, pasan- do de 4,6 a 3,3 muertes por 1.000 recién nacidos vivos. En el año 2004 se produce un repunte de la tasa que habrá que vigilar para ver si se mantiene o ha sido una variación aleatoria.

A nivel europeo, la tasa de Navarra se encuentra en el quintil inferior ${ }^{5}$ y se aproxima a los países que presentaron las tasas más bajas en el año 2002: Islandia $(2,3)$ y Suecia $(3,3)$.

\section{MORTALIDAD POR GRUPOS DE EDAD}

En la figura 2 se muestran las tasas específicas de mortalidad por edad en hombres y mujeres en los periodos 19951999 y 2000-2004 y las de España en el 2002. En todos los grupos de edad la mortalidad de los hombres es más alta que la

Tabla 1. Número de recién nacidos vivos, defunciones de menores de 1 año y tasas de mortalidad infantil por 1000 nacidos vivos. Navarra 1995-2004.

\begin{tabular}{cccc}
\hline Año & $\begin{array}{c}\text { Número recién } \\
\text { nacidos* }^{*}\end{array}$ & Muertes < 1año & $\begin{array}{c}\text { Tasa mortalidad } \\
\text { infantil }\end{array}$ \\
\hline 1995 & 4.627 & 31 & 6,70 \\
1996 & 4.830 & 26 & 5,38 \\
1997 & 4.967 & 19 & 3,83 \\
1998 & 4.952 & 27 & 5,45 \\
1999 & 5.122 & 10 & 1,95 \\
2000 & 5.262 & 21 & 3,99 \\
2001 & 5.710 & 17 & 2,98 \\
2002 & 5.802 & 15 & 2,59 \\
2003 & 6.180 & 14 & 2,27 \\
2004 & 6.293 & 29 & 4,61 \\
\hline
\end{tabular}

*Fuente: Instituto de Estadística de Navarra. 
de las mujeres. Las ratios de las tasas de mortalidad entre hombres y mujeres fueron en el periodo 2000-2004: 5-14 años $(2,1), 15-24$ años $(2,9), 25-34$ años $(2,6)$, 3544 años (2,3), 45-54 años (2,2), 55-64 años $(2,7)$ y $65-74$ años $(2,5)$. Expresado en cifras absolutas y tomando el año 2004, fallecieron en Navarra 1.020 hombres menores de 75 años frente a 464 mujeres.

En ambos sexos y en todos los grupos de edad descendieron las tasas de mortalidad en 2000-2004 respecto al quinquenio anterior excepto en las mujeres de 15-24 años donde se observó una pequeño incremento que habrá que analizar con más detalle y observar su evolución en los próximos años. Señalar que uno de los grupos donde más desciende la mortalidad es en los jóvenes de 25-34 años, grupo poblacional que en la década de los 80 experimentó un crecimiento de su riesgo de muerte a expensas fundamentalmente del sida. A partir de 1996, con la entrada de los nuevos tratamientos antirretrovirales el número de muertes al año por sida descendió de 20 a 2 en esta franja de edad.

También como se desprende de la figura 2, sobre todo en las edades medias y avanzadas las tasas de mortalidad de Navarra son más bajas que las registradas en España, siendo las diferencias más altas entre las mujeres.

\section{MORTALIDAD POR GRANDES GRUPOS DE CAUSAS}

Las principales causas de muerte en Navarra son el cáncer y las enfermedades circulatorias; entre ambas contabilizan aproximadamente el $60 \%$ de todas las muertes en los hombres y mujeres. Este patrón se asemeja al observado en el con-

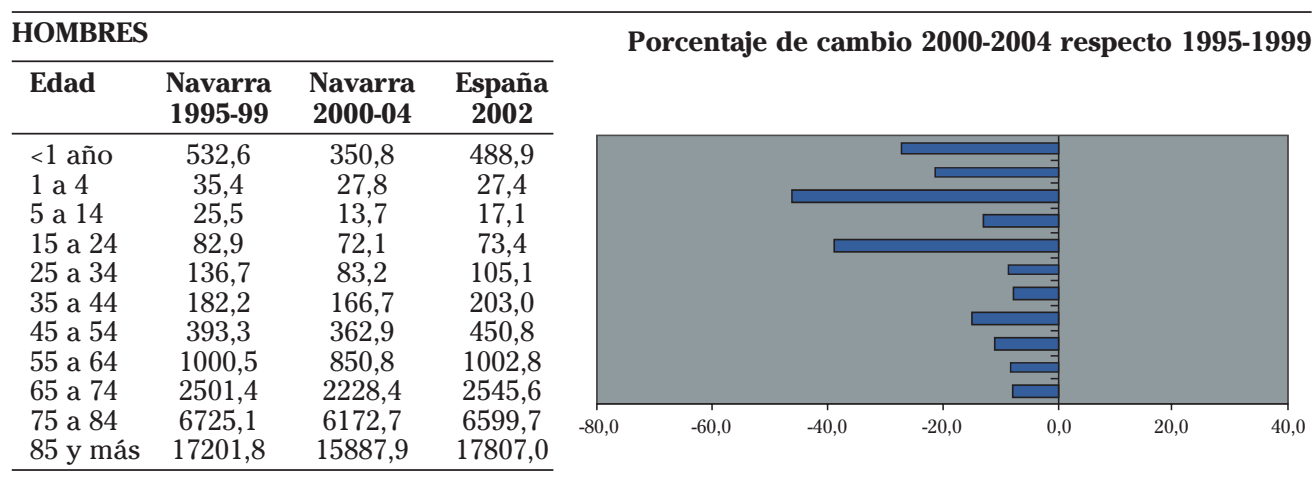

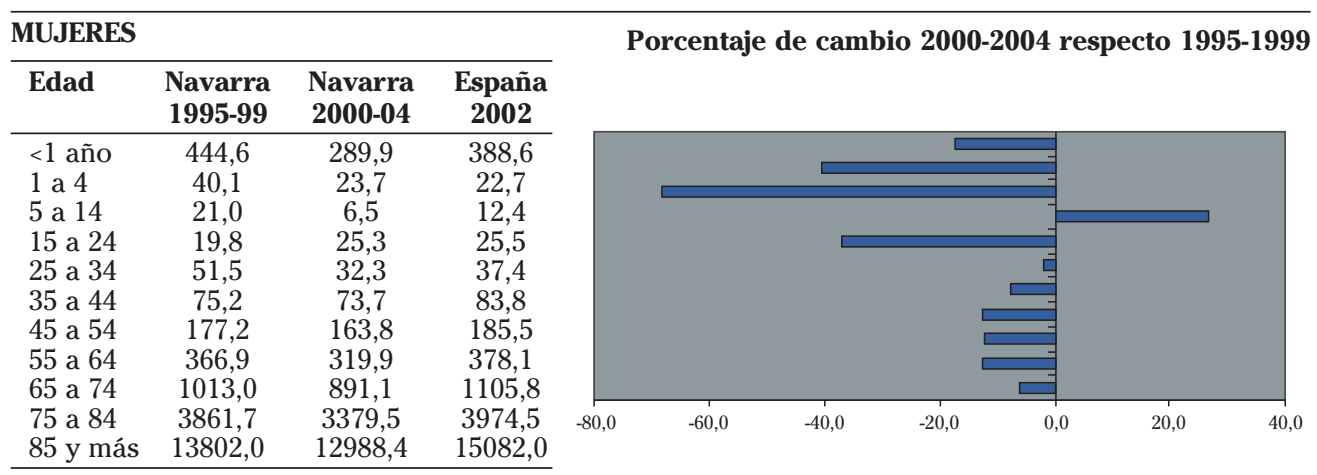

Figura 2. Tasas específicas de mortalidad por edad en Navarra y España. Tasas por 100.000. Porcentajes de cambio entre 2000-2004 vs 1995-1999 en Navarra. 
junto de los países de la UE-15 donde dos tercios de las muertes se deben a estas 2 causas. ${ }^{5}$

Entre los hombres, en el último periodo 2000-2004, el cáncer ocupa la primera causa de muerte en Navarra y las enfermedades circulatorias la segunda.

Cuando se compara la mortalidad de los hombres de Navarra con la de España, cabe señalar que las tasas de Navarra de todos los grandes grupos son más bajas que las de España excepto las tasas de mortalidad por causas externas (Tabla 2).

Entre las mujeres de Navarra, la mortalidad por enfermedades circulatorias continúa ocupando la primera posición, en segundo lugar destacan los tumores y en tercer y cuarto lugar la mortalidad por enfermedades respiratorias y las enfermedades del sistema nervioso. Cuando se

Tabla 2. Mortalidad por grandes grupos de causas en Navarra y España en el periodo 1995-2004 (orden decreciente del quinquenio 2000-2004 en Navarra). Tasas ajustadas por edad a la población europea por 100.000 .

\begin{tabular}{lcccc}
\hline Hombres & $\begin{array}{c}\text { Navarra } \\
\mathbf{1 9 9 5 - 9 9}\end{array}$ & $\begin{array}{c}\text { Navarra } \\
\mathbf{2 0 0 0 - 0 4}\end{array}$ & $\begin{array}{c}\text { España } \\
\mathbf{2 0 0 2}\end{array}$ & $\begin{array}{c}\text { Navarra respecto } \\
\text { de España }\end{array}$ \\
\hline Tumores & 252,0 & 233,9 & 252,1 & $-7,2$ \\
Enf. del sistema circulatorio & 237,0 & 195,4 & 226,8 & $-13,8$ \\
Enf. del sistema respiratorio & 90,1 & 80,8 & 95,3 & $-15,2$ \\
Causas externas de mortalidad & 58,1 & 53,5 & 51,0 & 4,9 \\
Enf. del sistema digestivo & 33,8 & 34,5 & 42,6 & $-19,0$ \\
Enf. del sistema nervioso y órg. sentidos & 20,5 & 19,6 & 20,4 & $-3,9$ \\
Enf. endocrinas, nutricionales y metabólicas & 15,4 & 14,6 & 17,6 & $-17,3$ \\
Enf. infecciosas y parasitarias & 16,1 & 12,3 & 16,4 & $-24,7$ \\
Causas mal definidas & 11,3 & 12,3 & 18,9 & $-34,8$ \\
Enf. del sistema genitourinario & 13,9 & 11,8 & 15,9 & $-26,0$ \\
Trastornos mentales y del comportamiento & 11,5 & 11,2 & 15,6 & $-28,5$ \\
Afecciones perinatales & 3,4 & 3,8 & 4,2 & $-8,6$ \\
Enf. sist. osteom y tej. conjuntivo & 3,4 & 3,5 & 4,2 & $-16,8$ \\
Malformaciones congénitas & 4,6 & 1,7 & 3,4 & $-51,3$ \\
Enfer. piel y tej. subcutáneo & 1,2 & 1,4 & 1,2 & 15,6 \\
Enf. sangre y órg. hematopoyéticos & 2,6 & 1,3 & 2,2 & $-41,1$ \\
Todas las causas & $\mathbf{7 7 4 , 8}$ & $\mathbf{6 9 1 , 6}$ & $\mathbf{7 8 7 , 7}$ & $\mathbf{- 1 2 , 2}$ \\
\hline
\end{tabular}

\begin{tabular}{lcccc}
\hline Mujeres & $\begin{array}{c}\text { Navarra } \\
\mathbf{1 9 9 5 - 9 9}\end{array}$ & $\begin{array}{c}\text { Navarra } \\
\mathbf{2 0 0 0 - 0 4}\end{array}$ & $\begin{array}{c}\text { España } \\
\mathbf{2 0 0 2}\end{array}$ & $\begin{array}{c}\text { Navarra respecto } \\
\text { de España }\end{array}$ \\
\hline Enf. del sistema circulatorio & 141,5 & 112,7 & 152,9 & $-26,3$ \\
Tumores & 117,0 & 108,6 & 112,3 & $-3,3$ \\
Enf. del sistema respiratorio & 36,2 & 33,3 & 37,1 & $-10,2$ \\
Enf. del sistema nervioso y órg. sentidos & 17,9 & 20,0 & 17,7 & 13,0 \\
Causa externas de mortalidad & 14,5 & 17,5 & 15,7 & 11,5 \\
Enf. del sistema digestivo & 17,8 & 16,4 & 21,9 & $-25,2$ \\
Enf. endocrinas, nutricionales y metabólicas & 13,1 & 12,8 & 16,7 & $-23,4$ \\
Trastornos mentales y del comportamiento & 12,3 & 10,2 & 15,7 & $-34,8$ \\
Causas mal definidas & 9,4 & 9,6 & 13,1 & $-27,0$ \\
Enf. del sistema genitourinario & 7,2 & 7,0 & 9,7 & $-28,0$ \\
Enf. infecciosas y parasitarias & 8,6 & 6,9 & 8,1 & $-15,1$ \\
Enf. sist. osteom y tej. conjuntivo & 5,8 & 4,7 & 5,5 & $-15,0$ \\
Malformaciones congénitas & 4,4 & 2,9 & 3,2 & $-8,5$ \\
Afecciones perinatales & 2,0 & 2,7 & 3,1 & $-14,4$ \\
Enfer.piel y tej. subcutáneo & 1,5 & 1,5 & 1,5 & 2,2 \\
Enf. sangre y órg. hematopoyéticos & 1,6 & 1,3 & 1,8 & $-27,7$ \\
Embarazo, parto y puerperio & 0,0 & 0,1 & 0,1 & $-39,5$ \\
Todas las causas & $\mathbf{4 1 0 , 9}$ & $\mathbf{3 6 8 , 1}$ & $\mathbf{4 3 6 , 2}$ & $\mathbf{- 1 5 , 6}$ \\
\hline
\end{tabular}


comparan las tasas de mortalidad de las mujeres de Navarra con las de España, se constata que la mortalidad por enfermedades circulatorias es bastante más baja en Navarra, casi un 30\%. En el resto de causas las diferencias son mucho menos llamativas siendo, por ejemplo, las tasas de mortalidad por cáncer prácticamente idénticas (Tabla 2).

\section{AÑOS POTENCIALES DE VIDA PERDIDOS}

En la tabla 3 se presentan las causas de muerte que producen mayor número de años potenciales de vida perdidos entre los hombres y las mujeres. Señalar en pri- mer lugar que la tasa de APVP es 2,2 veces más alta entre los hombres que en las mujeres. Los accidentes de tráfico, el cáncer de pulmón y el infarto agudo de miocardio ocupan los 3 primeros lugares como causas de mortalidad prematura entre los hombres, sumando entre ellas el $29,4 \%$ de los APVP. Como dato positivo merece señalar que las muertes prematuras por estas 3 causas han descendido en el último quinquenio (Tabla 3).

Entre las mujeres, también las muertes por accidentes de tráfico ocupan el primer lugar como causantes de APVP, mientras que la segunda posición la ocupan las muertes por afecciones originadas en el

Tabla 3. Defunciones anuales, edad media. Tasas estandarizadas a la población europea de Años Potenciales de Vida Perdidos de 0-70 años (TasaeAPVP) y Proporcional de la TasaeAPVP. Relación de las 15 causas en orden decreciente según la importancia relativa de la tasa estandarizada de APVP en 2000-04 en hombres y mujeres.

\begin{tabular}{|c|c|c|c|c|c|c|}
\hline & $\begin{array}{c}\text { Defunciones } \\
\text { anuales } \\
\text { totales }\end{array}$ & $\begin{array}{c}\text { Edad media } \\
\text { de todas las } \\
\text { muertes }\end{array}$ & $\begin{array}{r}\text { Tasae } \\
1995-99\end{array}$ & $\begin{array}{l}\text { APVP } \\
2000-04\end{array}$ & $\begin{array}{c}\% \text { total } \\
2000-04\end{array}$ & $\begin{array}{c}\text { \% cambio } \\
2000-04 \text { vs } \\
1995-99\end{array}$ \\
\hline \multicolumn{7}{|l|}{ Hombres } \\
\hline Accidentes de tráfico de vehículos de motor & 67 & 46,3 & 831,8 & 629,6 & 13,1 & $-32,1$ \\
\hline Tumor maligno de la tráquea, bronquios y del pulmón & 215 & 69,0 & 459,1 & 422,6 & 8,8 & $-8,6$ \\
\hline Infarto agudo de miocardio & 219 & 72,5 & 439,3 & 363,3 & 7,5 & $-20,9$ \\
\hline Afecciones originadas en el periodo perinatal & 7 & 0,0 & 257,6 & 288,8 & 6,0 & 10,8 \\
\hline Suicidio y lesiones autoinfligidas & 37 & 50,7 & 297,5 & 268,5 & 5,6 & $-10,8$ \\
\hline Sida & 14 & 41,5 & 314,9 & 129,8 & 2,7 & $-142,6$ \\
\hline Otras enfermedades del corazón & 115 & 76,0 & 116,8 & 126,2 & 2,6 & 7,4 \\
\hline Tumor maligno encéfalo & 24 & 61,7 & 122,5 & 125,7 & 2,6 & 2,6 \\
\hline Envenenamiento accidental psicofármacos y drogas & 10 & 34,4 & 130,6 & 118,7 & 2,5 & $-10,1$ \\
\hline Otras enf. del sistema nervioso y órganos sentidos & 41 & 70,0 & 134,5 & 111,9 & 2,3 & $-20,1$ \\
\hline Cirrosis y otras enfermedades crónicas del hígado & 38 & 65,8 & 106,3 & 110,8 & 2,3 & 4,1 \\
\hline Caídas accidentales & 23 & 65,1 & 62,2 & 92,5 & 1,9 & 32,8 \\
\hline Otros accidentes & 12 & 50,9 & 127,3 & 91,3 & 1,9 & $-39,3$ \\
\hline Enf. cerebrovasculares & 186 & 79,4 & 109,5 & 89,5 & 1,9 & $-22,4$ \\
\hline Todas las causas & 2647 & & $\mathbf{5 8 2 1 , 0}$ & 4819,8 & 61,6 & $-20,8$ \\
\hline
\end{tabular}

\begin{tabular}{|c|c|c|c|c|c|c|}
\hline \multicolumn{7}{|l|}{ Mujeres } \\
\hline Accidentes de tráfico de vehículos de motor & 23 & 52,5 & 211,6 & 205,6 & 9,4 & $-2,9$ \\
\hline Afecciones originadas en el periodo perinatal & 5 & 1,3 & 153,3 & 197,5 & 9,0 & 22,4 \\
\hline Tumor maligno de la mama & 74 & 70,3 & 263,8 & 181,5 & 8,3 & $-45,4$ \\
\hline Suicidio y lesiones autoinfligidas & 14 & 50,2 & 71,5 & 111,4 & 5,1 & 35,8 \\
\hline Tumor maligno de la tráquea, de los bronquios y del pulmón & 30 & 65,0 & 56,8 & 104,9 & 4,8 & 45,8 \\
\hline Otras enfermedades del sistema nervioso y órganos sentidos & 39 & 74,0 & 91,7 & 89,4 & 4,1 & $-2,5$ \\
\hline Tumor maligno encéfalo & 19 & 65,0 & 75,2 & 73,6 & 3,3 & $-2,2$ \\
\hline Malformaciones congénitas del sistema circulatorio & 2 & 10,4 & 136,2 & 73,6 & 3,3 & $-85,1$ \\
\hline Enf. cerebrovasculares & 250 & 84,3 & 51,0 & 66,1 & 3,0 & 22,9 \\
\hline Otras malformac. deformidades y anomalías cromosómicas & 3 & 31,4 & 130,7 & 65,7 & 3,0 & $-99,0$ \\
\hline Tumor maligno del colon & 56 & 76,4 & 52,1 & 63,8 & 2,9 & 18,3 \\
\hline Otras enfermedades del corazón & 133 & 82,6 & 70,4 & 50,2 & 2,3 & $-40,2$ \\
\hline Tumor maligno del ovario & 28 & 70,9 & 57,5 & 49,5 & 2,3 & $-16,2$ \\
\hline Sida & 4 & 40,2 & 132,2 & 41,3 & 1,9 & $-220,3$ \\
\hline Leucemia & 17 & 72,6 & 36,5 & 40,3 & 1,8 & 9,4 \\
\hline Todas las causas & 2364 & & 2653,0 & 2199,3 & 64,3 & $-20,6$ \\
\hline
\end{tabular}


periodo perinatal que se concentran en los menores de un año y en tercer lugar el cáncer de mama. Lo más llamativo en el último quinquenio es el fuerte descenso de la mortalidad prematura por cáncer de mama en relación, en buena medida, con el programa de screening.

\section{ENFERMEDADES DEL APARATO CIRCULATORIO}

Las muertes de causa cardiovascular representan el 29 y $36 \%$ del total de las muertes en los hombres y mujeres y el $15 \mathrm{y}$ 9\% de los APVP, respectivamente (datos no presentados) durante el periodo 2000-2004. En el último quinquenio las tasas de mortalidad por enfermedades cardiovasculares han disminuido un $17 \%$ en los hombres y un $19 \%$ en las mujeres y también las tasas de APVP, un $16 \%$ en los hombres y un $22 \%$ en las mujeres. El fuerte descenso de las tasas ha hecho que a pesar del aumento de población y del envejecimiento hayan descendido el número de fallecimientos por estas enfermedades (Tablas 4 y 5 ).

Tabla 4. Mortalidad por grupos de causas en Navarra en el periodo 1995-2004. Número medio de defunciones al año, mortalidad proporcional sobre el total de defunciones, tasas ajustadas por edad a la población de Navarra por 100.000 (TAN) y porcentaje de cambio de las tasas de 2000-2004 respecto 1995-1999. Hombres.

\begin{tabular}{|c|c|c|c|c|c|c|c|}
\hline & \multicolumn{2}{|c|}{ Número anual } & \multicolumn{2}{|c|}{$\%$ sobre total } & \multicolumn{2}{|c|}{ TAN } & \multirow{2}{*}{$\begin{array}{l}\text { \% cambio } \\
\text { tasas }\end{array}$} \\
\hline & 1995-99 & 2000-04 & 1995-99 & 2000-04 & 1995-99 & 200004 & \\
\hline Todas las causas & 2570 & 2648 & 100 & 100 & 1510 & 1365 & -10 \\
\hline Enfermedades infecciosas y parasitarias & $\mathbf{5 0}$ & 45 & 2 & 2 & 25 & 21 & -15 \\
\hline Enf. Infecciosas intestinales & 3 & 3 & 0,1 & 0,1 & 1,8 & 2,1 & 14,8 \\
\hline Tuberculosis y sus efectos tardíos & 3 & 3 & 0,1 & 0,1 & 1,8 & 1,4 & $-25,3$ \\
\hline Infecciones meningocócicas & 1 & 1 & 0,0 & 0,0 & 0,2 & 0,2 & $-11,4$ \\
\hline Septicemia & 11 & 18 & 0,4 & 0,7 & 7,7 & 9,9 & 29,9 \\
\hline Hepatitis vírica & 2 & 4 & 0,1 & 0,1 & 1,0 & 1,5 & 51,4 \\
\hline Sida & 27 & 14 & 1,0 & 0,5 & 10,0 & 4,6 & $-54,0$ \\
\hline VIH (portador. evidencias de laboratorio...) & 0 & 0 & 0,0 & 0,0 & 0,1 & 0,0 & - \\
\hline Resto de enfermedades infecciosas y parasitarias & 4 & 3 & 0,2 & 0,1 & 2,3 & 1,6 & $-32,7$ \\
\hline Tumores & 815 & 858 & $\mathbf{3 1 , 7}$ & 32,4 & 426,7 & 401,4 & $-5,9$ \\
\hline Tumor maligno del labio, cavidad bucal y faringe & 23 & 21 & 0,9 & 0,8 & 9,9 & 8,3 & $-15,6$ \\
\hline Tumor maligno del esófago & 22 & 21 & 0,8 & 0,8 & 9,5 & 8,6 & $-8,9$ \\
\hline Tumor maligno del estómago & 64 & 55 & 2,5 & 2,1 & 33,9 & 25,7 & $-24,1$ \\
\hline Tumor maligno del colon & 59 & 66 & 2,3 & 2,5 & 33,3 & 32,2 & $-3,5$ \\
\hline Tumor maligno del recto, rectosigmoide y ano & 23 & 26 & 0,9 & 1,0 & 12,4 & 12,4 & $-0,1$ \\
\hline Tumor maligno del hígado y vías biliares intrahepáticas & 34 & 37 & 1,3 & 1,4 & 17,8 & 17,3 & $-2,9$ \\
\hline Tumor maligno del páncreas & 35 & 43 & 1,4 & 1,6 & 16,9 & 19,7 & 16,7 \\
\hline Otros tumores malignos digestivos & 16 & 19 & 0,6 & 0,7 & 9,5 & 9,9 & 4,2 \\
\hline Tumor maligno de la laringe & 30 & 28 & 1,2 & 1,1 & 14,0 & 11,8 & $-15,7$ \\
\hline Tumor maligno de la tráquea, bronquios y del pulmón & 192 & 215 & 7,5 & 8,1 & 91,2 & 93,7 & 2,8 \\
\hline Otros tumores malignos respiratorios e intratorácicos & 7 & 5 & 0,3 & 0,2 & 3,3 & 2,0 & $-39,9$ \\
\hline Tumores malignos del hueso y cartílagos articulares & 2 & 2 & 0,1 & 0,1 & 0,9 & 1,0 & 19,9 \\
\hline Melanoma maligno de la piel & 6 & 8 & 0,2 & 0,3 & 2,6 & 3,7 & 41,8 \\
\hline Otros tumores de la piel y tejidos blandos & 10 & 8 & 0,4 & 0,3 & 5,6 & 4,2 & $-23,9$ \\
\hline Tumor maligno de la mama & 1 & 0 & 0,0 & 0,0 & 0,6 & 0,2 & $-69,3$ \\
\hline Tumor maligno de la próstata & 92 & 88 & 3,6 & 3,3 & 60,9 & 48,8 & $-19,9$ \\
\hline Otros tumores malignos de órganos genitales masculinos & 2 & 2 & 0,1 & 0,1 & 0,7 & 0,7 & $-9,9$ \\
\hline Tumor maligno del riñón, excepto pelvis renal & 16 & 17 & 0,6 & 0,6 & 8,1 & 7,9 & $-2,1$ \\
\hline Tumor maligno de la vejiga & 47 & 52 & 1,8 & 2,0 & 26,7 & 25,6 & $-4,0$ \\
\hline Otros tumores malignos de las vías urinarias & 4 & 2 & 0,1 & 0,1 & 2,2 & 0,9 & $-60,0$ \\
\hline Tumor maligno encéfalo & 24 & 24 & 0,9 & 0,9 & 10,3 & 9,9 & $-3,3$ \\
\hline Otros tumores malignos neurológicos y endocrínos & 3 & 4 & 0,1 & 0,2 & 1,3 & 2,0 & 45,5 \\
\hline $\begin{array}{l}\text { Tumor maligno de sitios mal definidos, } \\
\text { secundarios y no especif. }\end{array}$ & 39 & 47 & 1,5 & 1,8 & 20,8 & 23,4 & 12,7 \\
\hline $\begin{array}{l}\text { Otros tumores malignos del tejido linfático, } \\
\text { órg hematopoyéticos }\end{array}$ & 26 & 25 & 1,0 & 1,0 & 13,4 & 11,8 & $-11,8$ \\
\hline Leucemia & 22 & 24 & 0,8 & 0,9 & 10,9 & 11,0 & 0,1 \\
\hline Tumores benignos & 2 & 2 & 0,1 & 0,1 & 1,1 & 0,8 & $-24,9$ \\
\hline Síndrome mielodisplásico & 5 & 7 & 0,2 & 0,3 & 3,2 & 3,8 & 17,2 \\
\hline Otros tumores de comportamiento incierto o desconocido & 10 & 8 & 0,4 & 0,3 & 5,9 & 4,2 & $-28,7$ \\
\hline $\begin{array}{l}\text { Enf. sangre y órganos hematopoyéticos y inmunidad } \\
\text { Enfermedades de la sangre y de los }\end{array}$ & 9 & 5 & 0,3 & 0,2 & 6,2 & $\mathbf{3 , 3}$ & $-47,5$ \\
\hline órganos hematopoyéticos & 8 & 5 & 0,3 & 0,2 & 5,9 & 3,3 & $-44,5$ \\
\hline $\begin{array}{l}\text { Ciertos trastornos que afectan al mecanismo } \\
\text { de la inmunidad }\end{array}$ & 1 & & 0,0 & & 0,3 & . & \\
\hline
\end{tabular}




\begin{tabular}{|c|c|c|c|c|c|c|c|}
\hline & \multicolumn{2}{|c|}{ Número anual } & \multicolumn{2}{|c|}{$\%$ sobre total } & \multicolumn{2}{|c|}{ TAN } & \multirow{2}{*}{$\begin{array}{c}\% \text { cambio } \\
\text { tasas }\end{array}$} \\
\hline & 1995-99 & 2000-04 & 1995-99 & 2000-04 & 1995-99 & 200004 & \\
\hline Enfermedades endocrinas, nutricionales y metabólicas & s 51 & 57 & 2,0 & 2,2 & 31,5 & 31,4 & $-0,4$ \\
\hline Diabetes mellitus & 43 & 47 & 1,7 & 1,8 & 27,1 & 25,4 & $-6,3$ \\
\hline Otras enfermedades endocrinas, nutricinales y metabólicas & 8 & 10 & 0,3 & 0,4 & 4,4 & 6,0 & 35,9 \\
\hline Trastornos mentales y del comportamiento & 41 & 47 & 1,6 & 1,8 & 30,1 & 28,3 & $-5,9$ \\
\hline Trastornos mentales órganicos, senil y presenil & 35 & 41 & 1,4 & 1,5 & 27,4 & 25,6 & $-6,5$ \\
\hline Trastornos mentales debidos al uso de alcohol & 2 & 4 & 0,1 & 0,2 & 0,9 & 1,7 & 82,0 \\
\hline $\begin{array}{l}\text { Trastornos mentales debidos al uso de drogas } \\
\text { (drogodependenc.) }\end{array}$ & 0 & 0 & 0,0 & 0,0 & 0,2 & 0,1 & $-15,6$ \\
\hline Otros trastornos mentales y del comportamiento & 3 & 2 & 0,1 & 0,1 & 1,6 & 0,9 & $-43,3$ \\
\hline $\begin{array}{l}\text { Enfermedades del sistema nervioso, órganos } \\
\text { de los sentidos }\end{array}$ & 70 & 78 & 2,7 & $\mathbf{3 , 0}$ & 43,1 & 41,5 & $-3,6$ \\
\hline Meningitis (otras en 003) & 1 & 2 & 0,0 & 0,1 & 0,3 & 0,6 & 69,2 \\
\hline Enf. del Alzheimer & 33 & 36 & 1,3 & 1,3 & 22,8 & 21,1 & $-7,6$ \\
\hline $\begin{array}{l}\text { Otras enfermedades del sistema nervioso y } \\
\text { órganos de los sentidos }\end{array}$ & 36 & 41 & 1,4 & 1,5 & 19,9 & 19,9 & $-0,1$ \\
\hline Enfermedades del sistema circulatorio & 812 & 780 & 31,6 & 29,5 & 513,8 & 426,4 & $-17,0$ \\
\hline Enf. cardiacas reumáticas crónicas & 7 & 4 & 0,3 & 0,1 & 3,6 & 1,9 & $-46,9$ \\
\hline Enf. hipertensivas & 18 & 22 & 0,7 & 0,8 & 12,4 & 12,8 & 2,7 \\
\hline Infarto agudo de miocardio & 224 & 219 & 8,7 & 8,3 & 121,3 & 107,2 & $-11,6$ \\
\hline Otras enfermedades isquémicas del corazón & 88 & 88 & 3,4 & 3,3 & 56,1 & 48,7 & $-13,3$ \\
\hline Insuficiencia cardíaca & 101 & 85 & 3,9 & 3,2 & 77,5 & 55,1 & $-28,9$ \\
\hline Otras enfermedades del corazón & 93 & 115 & 3,6 & 4,4 & 57,2 & 61,7 & 7,8 \\
\hline Enf. cerebrovasculares & 202 & 186 & 7,9 & 7,0 & 135,1 & 105,5 & $-21,9$ \\
\hline Ateroesclerosis & 32 & 18 & 1,2 & 0,7 & 23,4 & 11,7 & $-50,2$ \\
\hline Otras enfermadades de los vasos sanguíneos & 48 & 42 & 1,9 & 1,6 & 27,1 & 22,0 & $-18,9$ \\
\hline Enfermedades del sistema respiratorio & 321 & 340 & 12,5 & 12,9 & 217,3 & 199,1 & $-8,4$ \\
\hline Influenza (gripe) & 5 & 2 & 0,2 & 0,1 & 3,4 & 1,3 & $-61,3$ \\
\hline Neumonía & 65 & 79 & 2,5 & 3,0 & 45,7 & 47,9 & 4,8 \\
\hline $\begin{array}{l}\text { Enf. crónicas de las vías respiratorias inferiores } \\
\text { (excepto asma) }\end{array}$ & 167 & 156 & 6,5 & 5,9 & 110,0 & 88,7 & $-19,4$ \\
\hline Asma & 3 & 1 & 0,1 & 0,1 & 1,8 & 0,5 & $-71,5$ \\
\hline Insuficiencia respiratoria & 9 & 16 & 0,4 & 0,6 & 7,2 & 9,4 & 29,8 \\
\hline Otras enfermedades del sistema respiratorio & 71 & 85 & 2,8 & 3,2 & 49,1 & 51,3 & 4,5 \\
\hline Enfermedades del sistema digestivo & 114 & 132 & 4,5 & $\mathbf{5 , 0}$ & 69,4 & 68,1 & $-1,9$ \\
\hline Úlcera de estómago, duodeno y yeyuno & 6 & 5 & 0,2 & 0,2 & 3,7 & 2,4 & $-36,9$ \\
\hline Enteritis y colitis no infecciosas & 2 & 2 & 0,1 & 0,1 & 0,9 & 1,0 & 8,9 \\
\hline Enf. vascular intestinal & 17 & 19 & 0,7 & 0,7 & 10,7 & 10,3 & $-3,8$ \\
\hline Cirrosis y otras enfermedades crónicas del hígado & 35 & 38 & 1,4 & 1,4 & 17,4 & 15,9 & $-8,6$ \\
\hline Otras enfermedades del sistema digestivo & 54 & 69 & 2,1 & 2,6 & 36,6 & 38,5 & 5,1 \\
\hline Enf. de la piel y del tejido subcutáneo & 4 & 6 & $\mathbf{0 , 2}$ & 0,2 & 3,1 & 3,7 & 18,9 \\
\hline Enf. del sistema osteomuscular y conjuntivo & 12 & 14 & 0,5 & 0,5 & 8,7 & 8,5 & $-2,2$ \\
\hline Artritis reumatoide y osteoartrosis & 2 & 1 & 0,1 & 0,0 & 0,9 & 0,5 & $-47,4$ \\
\hline Osteoporosis y fractura patológica & 7 & 10 & 0,3 & 0,4 & 5,7 & 6,4 & 13,2 \\
\hline $\begin{array}{l}\text { Otras enfermedades del sistema osteomuscular } \\
\text { y del tejido conj. }\end{array}$ & 3 & 3 & 0,1 & 0,1 & 2,2 & 1,6 & $-24,5$ \\
\hline Enfermedades del sistema genitourinario & 49 & 48 & 1,9 & 1,8 & 33,2 & 28,1 & $-15,3$ \\
\hline Enf. del riñón y del uréter & 41 & 38 & 1,6 & 1,4 & 27,3 & 21,9 & $-19,7$ \\
\hline Enf. de los genitales masculinos & 1 & 3 & 0,0 & 0,1 & 1,0 & 1,6 & 67,8 \\
\hline Otras enfermedades del sistema genitourinario & 7 & 8 & 0,3 & 0,3 & 4,9 & 4,5 & $-7,4$ \\
\hline Afecciones originadas en el periodo perinatal & 5 & 7 & $\mathbf{0 , 2}$ & 0,3 & 1,9 & 2,2 & 12,1 \\
\hline Malformaciones congénitas, deformidades y anomalías & Is 8 & 4 & $\mathbf{0 , 3}$ & $\mathbf{0 , 1}$ & 2,9 & 1,3 & $-55,5$ \\
\hline Malformaciones congénitasdel sistema nervioso & 1 & 0 & 0,0 & 0,0 & 0,3 & 0,1 & $-78,4$ \\
\hline Malformaciones congénitas del sistema circulatorio & 3 & 2 & 0,1 & 0,1 & 0,9 & 0,7 & $-26,0$ \\
\hline $\begin{array}{l}\text { Otras malformaciones congénitas deformidades y } \\
\text { anomalías cromosómicas }\end{array}$ & 5 & 1 & 0,2 & 0,1 & 1,7 & 0,5 & $-67,8$ \\
\hline $\begin{array}{l}\text { Síntomas, signos y hallazgos anormales } \\
\text { clínicos y de laborat. }\end{array}$ & 37 & $\mathbf{5 0}$ & 1,4 & 1,9 & 28,3 & 31,6 & 11,6 \\
\hline $\begin{array}{l}\text { Paro cardíaco, muerte sin asistencia y } \\
\text { otra causa desconocida }\end{array}$ & 14 & 28 & 0,6 & 1,1 & 9,8 & 17,5 & 78,5 \\
\hline Senilidad & 15 & 12 & 0,6 & 0,5 & 13,5 & 9,2 & $-31,6$ \\
\hline Muerte súbita infantil & 1 & 1 & 0,0 & 0,0 & 0,3 & 0,3 & 9,3 \\
\hline $\begin{array}{l}\text { Resto de síntomas, signos y estados morbosos } \\
\text { mal definidos }\end{array}$ & 7 & 8 & 0,3 & 0,3 & 4,7 & 4,5 & $-4,0$ \\
\hline
\end{tabular}




\begin{tabular}{|c|c|c|c|c|c|c|c|}
\hline & \multicolumn{2}{|c|}{ Número anual } & \multicolumn{2}{|c|}{ \% sobre total } & \multicolumn{2}{|c|}{ TAN } & \multirow{2}{*}{$\begin{array}{c}\% \text { cambio } \\
\text { tasas }\end{array}$} \\
\hline & 1995-99 & 2000-04 & 1995-99 & 2000-04 & 1995-99 & 200004 & \\
\hline Causas externas de mortalidad & 171 & 175 & 6,6 & 6,6 & 69,4 & 68,7 & $-1,0$ \\
\hline Accidentes de tráfico de vehículos de motor & 77 & 67 & 3,0 & 2,5 & 29,8 & 25,2 & $-15,4$ \\
\hline Otros accidentes de transporte & 3 & 3 & 0,1 & 0,1 & 1,3 & 1,0 & $-25,9$ \\
\hline Caídas accidentales & 13 & 23 & 0,5 & 0,9 & 6,6 & 10,8 & 63,4 \\
\hline Ahogamiento, sumersión y sofocación & 7 & 11 & 0,3 & 0,4 & 3,1 & 5,0 & 60,4 \\
\hline Accidentes por fuego, humo y sustancias calientes & 2 & 1 & 0,1 & 0,0 & 0,9 & 0,6 & $-33,9$ \\
\hline $\begin{array}{l}\text { Envenenamiento accidental por psicofármacos y } \\
\text { drogas de abuso }\end{array}$ & & & & & 3,5 & 3,4 & $-3,7$ \\
\hline $\begin{array}{l}\text { Arogas de abuso } \\
\text { Otros envenamientos accidentales }\end{array}$ & $\begin{array}{l}10 \\
2\end{array}$ & $\begin{array}{l}10 \\
2\end{array}$ & $\begin{array}{l}0,4 \\
0,1\end{array}$ & $\begin{array}{l}0,4 \\
0,1\end{array}$ & 0,6 & $\begin{array}{l}0,4 \\
0,9\end{array}$ & 43,0 \\
\hline Otros accidentes & 16 & 12 & 0,6 & 0,5 & 6,9 & 4,5 & $-34,0$ \\
\hline Suicidio y lesiones autoinfligidas & 35 & 37 & 1,4 & 1,4 & 14,4 & 14,2 & $-1,0$ \\
\hline Agresiones (homicidios) & 2 & 4 & 0,1 & 0,2 & 0,8 & 1,4 & 87,2 \\
\hline Eventos de intención no determinada & 0 & 1 & 0,0 & 0,0 & 0,1 & 0,3 & 255,5 \\
\hline Complicaciones de la atención médica y quirúrgica & 2 & 2 & 0,1 & 0,1 & 1,0 & 0,9 & $-9,9$ \\
\hline Otras causas externas y sus efectos tardíos & 1 & 1 & 0,0 & 0,1 & 0,4 & 0,5 & 23,4 \\
\hline
\end{tabular}

Tabla 5. Mortalidad por grupos de causas en Navarra en el periodo 1995-2004. Número medio de defunciones al año, mortalidad proporcional sobre el total de defunciones, tasas ajustadas por edad a la población de Navarra por 100.000 (TAN) y porcentaje de cambio de las tasas de 2000-2004 respecto 1995-1999. Mujeres.

\begin{tabular}{|c|c|c|c|c|c|c|c|}
\hline & \multicolumn{2}{|c|}{ Número anual } & \multicolumn{2}{|c|}{$\%$ sobre total } & \multicolumn{2}{|c|}{ TAN } & \multirow{2}{*}{$\begin{array}{c}\% \text { cambio } \\
\text { tasas }\end{array}$} \\
\hline & 1995-99 & 2000-04 & 1995-99 & 2000-04 & 1995-99 & 200004 & \\
\hline Todas las causas & 2215 & 2364 & 100,0 & 100,0 & 895,6 & 810,7 & $-9,5$ \\
\hline Enfermedades infecciosas y parasitarias & 36 & 40 & 1,6 & 1,7 & 14,3 & 13,7 & $-4,4$ \\
\hline Enf. Infecciosas intestinales & 3 & 3 & 0,1 & 0,1 & 1,8 & 2,1 & 14,8 \\
\hline Tuberculosis y sus efectos tardíos & 3 & 3 & 0,1 & 0,1 & 1,0 & 1,0 & $-3,0$ \\
\hline Infecciones meningocócicas & 2 & 0 & 0,1 & 0,0 & 0,7 & 0,1 & $-78,8$ \\
\hline Septicemia & 14 & 19 & 0,6 & 0,8 & 5,7 & 6,5 & 15,2 \\
\hline Hepatitis vírica & 1 & 3 & 0,1 & 0,1 & 0,5 & 1,0 & 99,0 \\
\hline Sida & 9 & 4 & 0,4 & 0,2 & 3,5 & 1,4 & $-60,5$ \\
\hline VIH (portador. evidencias de laboratorio...) & 0 & 0 & 0,0 & 0,0 & 0,0 & 0,0 & \\
\hline Resto de enfermedades infeciosas y parasitarias & 4 & 5 & 0,2 & 0,2 & 1,4 & 1,8 & 29,2 \\
\hline Tumores & 502 & 531 & 22,7 & 22,4 & 195,9 & 183,5 & $-6,3$ \\
\hline Tumor maligno del labio, cavidad bucal y faringe & 5 & 5 & 0,2 & 0,2 & 2,1 & 1,8 & $-15,1$ \\
\hline Tumor maligno del esófago & 3 & 4 & 0,2 & 0,2 & 1,3 & 1,3 & $-4,1$ \\
\hline Tumor maligno del estómago & 41 & 35 & 1,8 & 1,5 & 16,1 & 12,2 & $-24,5$ \\
\hline Tumor maligno del colon & 50 & 56 & 2,3 & 2,4 & 19,8 & 19,4 & $-1,5$ \\
\hline Tumor maligno del recto, rectosigmoide y ano & 14 & 20 & 0,6 & 0,9 & 5,3 & 7,0 & 31,9 \\
\hline Tumor maligno del hígado y vías biliares intrahepáticas & 20 & 22 & 0,9 & 0,9 & 7,9 & 7,5 & $-4,4$ \\
\hline Tumor maligno del páncreas & 32 & 35 & 1,5 & 1,5 & 12,5 & 11,9 & $-4,5$ \\
\hline Otros tumores malignos digestivos & 26 & 27 & 1,2 & 1,2 & 10,3 & 9,4 & $-8,6$ \\
\hline Tumor maligno de la laringe & 1 & 0 & 0,0 & 0,0 & 0,4 & 0,1 & $-65,1$ \\
\hline Tumor maligno de la tráquea, bronquios y pulmón & 22 & 30 & 1,0 & 1,3 & 8,7 & 10,3 & 18,6 \\
\hline Otros tumores malignos respiratorios e intratorácicos & 3 & 4 & 0,2 & 0,2 & 1,3 & 1,5 & 15,0 \\
\hline Tumores malignos del hueso y cartílagos articulares & 2 & 1 & 0,1 & 0,0 & 0,8 & 0,3 & $-64,1$ \\
\hline Melanoma maligno de la piel & 4 & 6 & 0,2 & 0,2 & 1,7 & 2,0 & 14,1 \\
\hline Otros tumores de la piel y tejidos blandos & 6 & 6 & 0,3 & 0,3 & 2,3 & 2,2 & $-2,1$ \\
\hline Tumor maligno de la mama & 81 & 74 & 3,6 & 3,1 & 31,2 & 25,7 & $-17,7$ \\
\hline Tumor maligno del cuello del útero & 3 & 5 & 0,1 & 0,2 & 1,1 & 1,9 & 72,0 \\
\hline Tumor maligno de otras partes del útero & 19 & 15 & 0,9 & 0,6 & 7,5 & 5,2 & $-30,3$ \\
\hline Tumor maligno del ovario & 22 & 28 & 1,0 & 1,2 & 8,6 & 9,7 & 12,3 \\
\hline Otros tumores malignos de órganos genitales femeninos & 7 & 5 & 0,3 & 0,2 & 2,9 & 1,8 & $-37,4$ \\
\hline Tumor maligno del riñón, excepto pelvis renal & 7 & 11 & 0,3 & 0,5 & 2,9 & 3,8 & 32,6 \\
\hline Tumor maligno de la vejiga & 11 & 12 & 0,5 & 0,5 & 4,3 & 4,0 & $-6,7$ \\
\hline Otros tumores malignos de las vías urinarias & 1 & 1 & 0,0 & 0,1 & 0,3 & 0,4 & 34,1 \\
\hline Tumor maligno encéfalo & 18 & 19 & 0,8 & 0,8 & 6,7 & 6,7 & 0,4 \\
\hline Otros tumores malignos neurológicos y endocrínos & 6 & 4 & 0,3 & 0,2 & 2,2 & 1,3 & $-39,2$ \\
\hline $\begin{array}{l}\text { Tumor maligno de sitios mal definidos, } \\
\text { secundarios y no esp. }\end{array}$ & 34 & 34 & 1,5 & 1,5 & 13,4 & 11,9 & $-11,1$ \\
\hline Otros tumores malignos del tejido linfático, & & & & & & & \\
\hline órg. hematopoyéticos & 29 & 29 & 1,3 & 1,2 & 11,1 & 10,0 & $-10,2$ \\
\hline Leucemia & 17 & 17 & 0,7 & 0,7 & 6,5 & 5,8 & $-10,5$ \\
\hline Tumores benignos & 3 & 7 & 0,1 & 0,3 & 1,2 & 2,5 & 116,8 \\
\hline Síndrome mielodisplásico & 3 & 4 & 0,1 & 0,2 & 1,2 & 1,5 & 22,7 \\
\hline Otros tumores de comportamiento & & & & & & & \\
\hline incierto o desconocido & 12 & 13 & 0,5 & 0,5 & 4,6 & 4,3 & $-6,4$ \\
\hline
\end{tabular}




\begin{tabular}{|c|c|c|c|c|c|c|c|}
\hline & \multicolumn{2}{|c|}{ Número anual } & \multicolumn{2}{|c|}{$\%$ sobre total } & \multicolumn{2}{|c|}{ TAN } & \multirow{2}{*}{$\begin{array}{c}\% \text { cambio } \\
\text { tasas }\end{array}$} \\
\hline & 1995-99 & 2000-04 & 1995-99 & 2000-04 & 1995-99 & 200004 & \\
\hline \multicolumn{8}{|l|}{ Enfermedades de la sangre y de los } \\
\hline órganos hematopoyéticos & 9 & 9 & $\mathbf{0 , 4}$ & $\mathbf{0 , 4}$ & 3,7 & $\mathbf{3 , 0}$ & $-19,0$ \\
\hline \multicolumn{8}{|l|}{ Enfermedades de la sangre y de los } \\
\hline órganos hematopoyéticos & 9 & 9 & 0,4 & 0,4 & 3,6 & 2,9 & $-17,2$ \\
\hline \multicolumn{8}{|l|}{ Ciertos trastornos que afectan al } \\
\hline Enfermedades endocrinas, nutricionales y metabólicas & 74 & $\begin{array}{c}0 \\
89\end{array}$ & $\begin{array}{l}0,0 \\
\mathbf{3 , 3}\end{array}$ & $\begin{array}{l}0,0 \\
3,8\end{array}$ & $\begin{array}{c}0,2 \\
\mathbf{2 9 , 9}\end{array}$ & $\begin{array}{c}0,1 \\
\mathbf{3 0 , 5}\end{array}$ & $\begin{array}{c}-57,9 \\
2,0\end{array}$ \\
\hline Diabetes mellitus & 61 & 71 & 2,8 & 3,0 & 24,8 & 24,3 & $-2,2$ \\
\hline \multicolumn{8}{|l|}{ Otras enfermedades endocrinas, } \\
\hline nutricionales y metabólicas & 13 & 18 & 0,6 & 0,8 & 5,1 & 6,2 & 22,7 \\
\hline Trastornos mentales y del comportamiento & 82 & 87 & 3,7 & 3,7 & 33,9 & 29,5 & $-12,9$ \\
\hline Trastornos mentales orgánicos, senil y presenil & 77 & 85 & 3,5 & 3,6 & 32,3 & 28,8 & $-10,8$ \\
\hline Trastornos mentales debidos al uso de alcohol & 1 & 0 & 0,0 & 0,0 & 0,2 & 0,1 & $-39,6$ \\
\hline \multicolumn{8}{|l|}{ Trastornos mentales debidos al uso de drogas } \\
\hline (drogodepencias) & 0 & 0 & 0,0 & 0,0 & 0,1 & 0,0 & $-100,0$ \\
\hline Otros trastornos mentales y del comportamiento & 3 & 2 & 0,2 & 0,1 & 1,4 & 0,6 & $-54,7$ \\
\hline \multicolumn{8}{|l|}{ Enfermedades del sistema nervioso y } \\
\hline órganos de los sentidos & 100 & 138 & 4,5 & 5,8 & 40,1 & 47,3 & 17,7 \\
\hline Meningitis (otras en 003) & 1 & 1 & 0,0 & 0,0 & 0,3 & 0,4 & 8,3 \\
\hline Enf. del Alzheimer & 68 & 98 & 3,1 & 4,2 & 27,8 & 33,6 & 20,9 \\
\hline Otras enfermedades del sistema nerivioso y & & & & & & & \\
\hline órganos sentidos & 31 & 39 & 1,4 & 1,6 & 12,0 & 13,3 & 10,7 \\
\hline Enfermedades del sistema circulatorio & 872 & 844 & 39,4 & $\mathbf{3 5 , 7}$ & $\mathbf{3 5 6 , 9}$ & 288,7 & $-19,1$ \\
\hline Enf. cardiacas reumáticas crónicas & 14 & 12 & 0,6 & 0,5 & 5,4 & 4,2 & $-22,2$ \\
\hline Enf. hipertensivas & 36 & 44 & 1,6 & 1,9 & 14,6 & 15,1 & 3,6 \\
\hline Infarto agudo de miocardio & 136 & 137 & 6,1 & 5,8 & 54,7 & 47,1 & $-13,9$ \\
\hline Otras enfermedades isquémicas del corazón & 77 & 59 & 3,5 & 2,5 & 31,8 & 20,2 & $-36,5$ \\
\hline Insuficiencia cardíaca & 187 & 168 & 8,4 & 7,1 & 78,1 & 57,3 & $-26,6$ \\
\hline Otras enfermedades del corazón & 101 & 133 & 4,6 & 5,6 & 40,9 & 45,8 & 11,8 \\
\hline Enf. cerebrovasculares & 265 & 250 & 12,0 & 10,6 & 108,4 & 85,3 & $-21,3$ \\
\hline Ateroesclerosis & 33 & 18 & 1,5 & 0,8 & 13,9 & 6,2 & $-55,5$ \\
\hline Otras enfermadades de los vasos sanguíneos & 22 & 22 & 1,0 & 0,9 & 9,0 & 7,5 & $-17,0$ \\
\hline Enfermedades del sistema respiratorio & 225 & 256 & 10,2 & 10,8 & 92,3 & 87,3 & $-5,4$ \\
\hline Influenza (gripe) & 7 & 3 & 0,3 & 0,1 & 2,8 & 1,0 & $-66,3$ \\
\hline Neumonía & 68 & 75 & 3,1 & 3,2 & 27,9 & 25,5 & $-8,5$ \\
\hline Enf. crónicas de las vías respiratorias (excepto asma) & 55 & 43 & 2,5 & 1,8 & 22,4 & 14,7 & $-34,5$ \\
\hline Asma & 9 & 7 & 0,4 & 0,3 & 3,6 & 2,5 & $-31,8$ \\
\hline Insuficiencia respiratoria & 11 & 21 & 0,5 & 0,9 & 4,7 & 7,3 & 56,6 \\
\hline Otras enfermedades del sistema respiratorio & 75 & 106 & 3,4 & 4,5 & 30,9 & 36,4 & 17,8 \\
\hline Enfermedades del sistema digestivo & 103 & 111 & 4,7 & 4,7 & 41,9 & 38,2 & $-8,9$ \\
\hline Úlcera de estómago, duodeno y yeyuno & 5 & 4 & 0,2 & 0,2 & 2,2 & 1,4 & $-32,9$ \\
\hline Enteritis y colitis no infecciosas & 1 & 1 & 0,1 & 0,0 & 0,6 & 0,3 & $-50,7$ \\
\hline Enf. vascular intestinal & 18 & 22 & 0,8 & 0,9 & 7,2 & 7,4 & 3,0 \\
\hline Cirrosis y otras enfermedades crónicas del hígado & 12 & 11 & 0,6 & 0,5 & 4,8 & 3,9 & $-17,8$ \\
\hline Otras enfermedades del sistema digestivo & 67 & 73 & 3,0 & 3,1 & 27,2 & 25,1 & $-7,6$ \\
\hline Enf. de la piel y del tejido subcutáneo & 10 & 13 & $\mathbf{0 , 5}$ & 0,6 & 4,2 & 4,4 & 6,5 \\
\hline Enf. del sistema osteomuscular y del tejido conjuntivo & 36 & 35 & 1,6 & 1,5 & 15,1 & 12,1 & $-20,1$ \\
\hline Artritis reumatoide y osteoartrosis & 3 & 2 & 0,1 & 0,1 & 1,3 & 0,8 & $-40,4$ \\
\hline Osteoporósis y fractura patológica & 29 & 27 & 1,3 & 1,1 & 12,2 & 9,2 & $-24,7$ \\
\hline Otras enfermedades del sistema & & & & & & & \\
\hline osteomuscular y conjuntivo & 4 & 6 & 0,2 & 0,3 & 1,6 & 2,1 & 29,9 \\
\hline Enfermedades del sistema genitourinario & 42 & 49 & 1,9 & 2,1 & 17,1 & 16,9 & $-1,5$ \\
\hline Enf. del riñón y del uréter & 36 & 37 & 1,6 & 1,6 & 14,7 & 12,8 & $-13,0$ \\
\hline Enf. de los genitales masculinos & 0 & 0 & 0,0 & 0,0 & 0,0 & 0,0 & \\
\hline Otras enfermedades del sistema genitourinario & 6 & 12 & 0,3 & 0,5 & 2,3 & 4,0 & 72,6 \\
\hline Embarazo, parto y puerperio* & $\mathbf{0}$ & $\mathbf{0}$ & $\mathbf{0 , 0}$ & $\mathbf{0 , 0}$ & $\mathbf{0 , 0}$ & $\mathbf{0 , 1}$ & \\
\hline Afecciones originadas en el periodo perinatal & $\mathbf{3}$ & $\mathbf{5}$ & $\mathbf{0 , 1}$ & $\mathbf{0 , 2}$ & 1,1 & 1,5 & 33,3 \\
\hline Malformaciones congénitas, deformidades y anomalías & is 7 & 6 & $\mathbf{0 , 3}$ & $\mathbf{0 , 3}$ & 2,8 & 2,1 & $-24,9$ \\
\hline Malformaciones congénitas del sistema nervioso & 1 & 1 & 0,0 & 0,1 & 0,3 & 0,4 & 38,1 \\
\hline Malformaciones congénitas del sistema circulatorio & 3 & 2 & 0,1 & 0,1 & 1,1 & 0,7 & $-41,1$ \\
\hline Otras malformaciones congénitas y & & & & & & & \\
\hline anomalías cromosómicas & 4 & 3 & 0,2 & 0,1 & 1,4 & 1,0 & $-25,5$ \\
\hline Síntomas, signos y hallazgos anormales clínicos & & & & & & & \\
\hline y de laboratorio & 61 & 80 & 2,8 & 3,4 & 25,9 & 27,2 & 5,2 \\
\hline Paro cardíaco, muerte sin asistencia y & & & & & & & \\
\hline causa desconocida & 22 & 34 & 1,0 & 1,5 & 9,2 & 11,7 & 27,6 \\
\hline Senilidad & 32 & 30 & 1,4 & 1,3 & 13,5 & 10,1 & $-25,6$ \\
\hline Muerte súbita infantil & 0 & 0 & 0,0 & 0,0 & 0,1 & 0,1 & 72,9 \\
\hline Resto de síntomas, signos y estados & & & & & & & \\
\hline morbosos mal definidos & 7 & 16 & 0,3 & 0,7 & 3,1 & 5,3 & 71,9 \\
\hline
\end{tabular}




\begin{tabular}{|c|c|c|c|c|c|c|c|}
\hline & \multicolumn{2}{|c|}{ Número anual } & \multicolumn{2}{|c|}{$\%$ sobre total } & \multicolumn{2}{|c|}{ TAN } & \multirow{2}{*}{$\begin{array}{c}\text { \% cambio } \\
\text { tasas }\end{array}$} \\
\hline & 1995-99 & 2000-04 & 1995-99 & 2000-04 & 1995-99 & 200004 & \\
\hline Causas externas de mortalidad & 53 & 71 & 2,4 & $\mathbf{3 , 0}$ & 20,3 & 24,6 & 21,4 \\
\hline Accidentes de tráfico de vehículos de motor & 19 & 23 & 0,8 & 1,0 & 7,0 & 8,1 & 16,5 \\
\hline Otros accidentes de transporte & 1 & 1 & 0,1 & 0,0 & 0,4 & 0,3 & $-31,1$ \\
\hline Caídas accidentales & 10 & 17 & 0,5 & 0,7 & 4,3 & 5,7 & 34,9 \\
\hline Ahogamiento, sumersión y sofocación & 3 & 3 & 0,1 & 0,1 & 1,1 & 1,1 & $-1,4$ \\
\hline Accidentes por fuego, humo y sustancias calientes & 2 & 1 & 0,1 & 0,1 & 0,9 & 0,4 & $-53,1$ \\
\hline $\begin{array}{l}\text { Envenenamiento accidental psicofármacos, } \\
\text { drogas de abuso }\end{array}$ & 1 & 3 & 0,0 & 0,1 & 0,3 & 0,9 & 192,2 \\
\hline Otros envenamientos accidentales & 0 & 0 & 0,0 & 0,0 & 0,2 & 0,0 & $-100,0$ \\
\hline Otros accidentes & 3 & 4 & 0,1 & 0,2 & 1,3 & 1,5 & 17,3 \\
\hline Suicidio y lesiones autoinfligidas & 9 & 14 & 0,4 & 0,6 & 3,5 & 4,8 & 36,9 \\
\hline Agresiones (homicidios) & 1 & 2 & 0,1 & 0,1 & 0,4 & 0,6 & 27,1 \\
\hline Eventos de intención no determinada & 0 & 0 & 0,0 & 0,0 & 0,1 & 0,1 & 73,2 \\
\hline Complicaciones de la atención médica y quirúrgica & 2 & 3 & 0,1 & 0,1 & 0,9 & 1,0 & 13,4 \\
\hline Otras causas externas y sus efectos tardíos & 0 & 0 & 0,0 & 0,0 & 0,0 & 0,1 & \\
\hline
\end{tabular}

* 1 muerte en el quinquenio $2000-04$.

El descenso se ha producido en ambos sexos en la mortalidad por enfermedad cerebrovascular, enfermedad isquémica del corazón, insuficiencia cardiaca y arterioesclerosis, principales subgrupos del capítulo de enfermedades circulatorias. En Navarra, el descenso de la mortalidad por enfermedades cardiovasculares es en gran medida responsable del aumento de la esperanza de vida al nacer en la década de los 90 , tanto en hombres como en mujeres, si consideramos que son las patologías que más defunciones causan y que han disminuido de manera muy significativa en los últimos años.

Existe una gran disparidad dentro de la UE para la mortalidad por estas enfermedades y además hay que señalar que el patrón geográfico es diferente para la mortalidad por enfermedad isquémica del corazón y para la mortalidad por enfermedad cerebrovascular ${ }^{5}$. Navarra se encuentra entre las regiones de Europa con tasas muy bajas tanto para el conjunto de la mortalidad por enfermedades cardiovasculares como para la mortalidad por enfermedad isquémica del corazón e igualmente para la enfermedad cerebrovascular (Tabla 6). Francia y Suiza presenta las tasas más bajas y las tasas de Navarra son muy parecidas a las encontradas en esos países. La menor mortalidad en Navarra respecto a España se observa tanto para la mortalidad por enfermedad isquémica como para la mortalidad por enfermedad cerebrovascular (Tabla 6).

\section{CÁNCER}

En el último periodo, 2000-2004, las muertes por tumores representaban el $32,4 \%$ de todas las muertes entre los hombres y el $22,4 \%$ en las mujeres. Son también una importante causa mortalidad prematura, representando el 31 y $42 \%$ de los APVP, respectivamente.

La tasa de mortalidad por cáncer de los hombres de Navarra es un 7\% más baja que la de España y además se ha producido un descenso de la mortalidad por esta causa en los últimos años. El descenso de la mortalidad por cáncer ha afectado también a la mortalidad prematura o tasa de APVP, que ha descendido un $13 \%$ en los hombres (datos no presentados). El descenso de las tasas de mortalidad por tumores del aparato digestivo en prácticamente todas las localizaciones (excepto páncreas), y fundamentalmente el importante descenso de la mortalidad por cáncer de estómago, son en buena medida responsables de la buena evolución del indicador "mortalidad por cáncer" (Tabla 4). A nivel europeo la tasa de los hombres se encuentra en torno a la media (Tabla 6).

Entre las mujeres considerando conjuntamente todos los tipos de tumores, se observa un descenso de las tasas de $7 \%$ en el último quinquenio (Tabla 3) y lo mismo ocurre con la mortalidad prematura que ha descendido un $9,2 \%$ (datos no presentados). A nivel europeo, la tasa de mortalidad por cáncer de las mujeres de Navarra se encuentra entre las más bajas y se apro- 
Tabla 6. Mortalidad por determinadas causas de muerte en Navarra, España y UE-15. Tasas ajustadas a la población europea. Datos de 1998 y 1995-1999 en el caso de Navarra.

\begin{tabular}{|c|c|c|}
\hline & Varones & Mujeres \\
\hline \multicolumn{3}{|l|}{ Todas las causas } \\
\hline Navarra & 774,8 & 410,9 \\
\hline España & 865,0 & 478,9 \\
\hline Unión Europea & 890,0 & 529,0 \\
\hline Tasa más baja de la Unión Europea & 759 (Suiza) & 442,2 (Suiza) \\
\hline \multicolumn{3}{|l|}{ Enfermedades del sistema circulatorio } \\
\hline Navarra & 237,9 & 141,5 \\
\hline España & 274,7 & 187,0 \\
\hline Unión Europea & 337,7 & 212,8 \\
\hline Tasa más baja de la Unión Europea & 235,8 (Francia) & 137 (Francia) \\
\hline \multicolumn{3}{|l|}{ Enfermedades isquémicas del corazón } \\
\hline Navarra & 93,9 & 36,0 \\
\hline España & 102,9 & 45,3 \\
\hline Unión Europea & 151,2 & 73,7 \\
\hline Tasa más baja de la Unión Europea & 78,8 (Francia) & 32,4 (Francia) \\
\hline \multicolumn{3}{|l|}{ Enfermedades cerebrovasculares } \\
\hline Navarra & 56,8 & 42,7 \\
\hline España & 69,8 & 56,5 \\
\hline Unión Europea & 74,8 & 67,1 \\
\hline Tasa más baja de la Unión Europea & 45,2 (Suiza) & 34,6 (Suiza) \\
\hline \multicolumn{3}{|l|}{ Tumores } \\
\hline Navarra & 252,0 & 117,0 \\
\hline España & 263,1 & 116,4 \\
\hline Unión Europea & 258,7 & 144,5 \\
\hline Tasa más baja de la Unión Europea & 211,9 (Grecia) & 112,5 (Grecia) \\
\hline \multicolumn{3}{|l|}{ Cáncer colorrectal } \\
\hline Navarra & 25,1 & 13,7 \\
\hline España & 27,8 & 15,6 \\
\hline Unión Europea & 27,1 & 17,1 \\
\hline Tasa más baja de la Unión Europea & 12,7 (Grecia) & 11,9 (Italia) \\
\hline \multicolumn{3}{|l|}{ Cáncer de estómago } \\
\hline Navarra & 19,8 & 8,6 \\
\hline España & 17,0 & 7,3 \\
\hline Unión Europea & 15,1 & 7,1 \\
\hline Tasa más baja de la Unión Europea & 8 (Dinamarca) & 4,1 (Francia) \\
\hline \multicolumn{3}{|c|}{ Cáncer de laringe/tráquea/bronquios/pulmón } \\
\hline Navarra & 70,7 & 5,9 \\
\hline España & 78,9 & 6,5 \\
\hline Unión Europea & 72,8 & 15,9 \\
\hline Tasa más baja de la Unión Europea & 32,9 (Suecia) & 6,5 (España) \\
\hline \multicolumn{3}{|l|}{ Cáncer de mama } \\
\hline Navarra & & 22,2 \\
\hline España & & 22,2 \\
\hline Unión Europea & & 28,0 \\
\hline Tasa más baja de la Unión Europea & & 20,8 (Grecia) \\
\hline \multicolumn{3}{|l|}{ Accidentes de tráfico } \\
\hline Navarra & 27,9 & 6,7 \\
\hline España & 23,6 & 6,9 \\
\hline Unión Europea & 17,6 & 5,5 \\
\hline Tasa más baja de la Unión Europea & 8,9 (Reino Unido & 3 (Suecia, Reino Unido) \\
\hline \multicolumn{3}{|l|}{ Suicidios } \\
\hline Navarra & 11,6 & 2,9 \\
\hline España & 11,7 & 3,2 \\
\hline Unión Europea & 16,9 & 5,1 \\
\hline Tasa más baja de la Unión Europea & 7,9 (Portugal) & 2,2 (Portugal) \\
\hline
\end{tabular}


xima al país con tasa más baja que es Grecia (Tabla 6).

\section{Cáncer de pulmón}

Fallecen anualmente 215 hombres y 30 mujeres por cáncer de pulmón en Navarra. Entre los hombres, uno de cada 12 muere por esta causa, representando además una de cada 4 muertes por cáncer. Entre las mujeres, las muertes por cáncer de pulmón representan una de cada 20 muertes por cáncer (Tabla 4).

El cáncer de pulmón es el tumor que mayor tasa de mortalidad ha presentado entre los hombres de Navarra a lo largo de toda la década y ha continuado aumentando un 2,8\% en el periodo $2000-04$ respecto al periodo 1995-1999. Es, tras los accidentes de tráfico, la segunda causa de muerte prematura en los hombres y como dato esperanzador la tasa de APVP en menores de 70 años fue un 8\% menor en 2000-2004 que en 1995-1999 (Tabla 3). La tasa de mortalidad de Navarra $(70,7)$ se encuentra por debajo de la tasa global de la UE-15 $(78,9)$ y muy por encima de la de países como Suecia $(32,9)$, Noruega $(47,1)$ o Suiza $(53)$. En Navarra todavía no se ha alcanzado el punto más alto de la curva de mortalidad para este tumor en los hombres mientras numerosos países de la Unión Europea o los Estados Unidos muestran tasas de mortalidad claramente descendentes desde hace bastantes años ${ }^{6-10}$. En Europa las tasas de mortalidad por cáncer de pulmón comenzaron a descender desde finales de los 70 en los países del norte y oeste (Austria, Bélgica, países nórdicos o Reino Unido), desde finales de los 80 en el conjunto de los países mediterráneos (Francia, Grecia, Portugal, Italia y España), mientras que los países del este (Hungría, Polonia o Checoslovaquia) presentan tasas crecientes ${ }^{11}$. En Cataluña, en el periodo 1989-1998 la mortalidad por cáncer de pulmón presentó una tendencia descendente con un porcentaje de descenso anual de $0,8^{12}$.

Los indicadores relativos a la mortalidad por cáncer de pulmón en las mujeres registran incrementos importantes en el periodo 2000-2004 que se reflejan sobre todo en la mortalidad prematura que ha aumentado un $45,8 \%$ y menos en la tasa global de mortalidad que ha aumentado un $18,6 \%$ (Tablas 3 y 5 ), lo que traduce que el exceso de mortalidad por este cáncer. Sólo afecta a las mujeres jóvenes y no a las mayores que prácticamente no han fumado. Las estadísticas de las mujeres jóvenes señalan el comienzo de la epidemia de cáncer de pulmón entre las mujeres de Navarra. Todavía, sin embargo, continúa siendo mucho más baja que la de la mayoría de los países de la Unión Europea ${ }^{3-5}$ encontrándose nuestra región en la parte ascendente de la curva de mortalidad, reflejo de la tardía introducción del tabaquismo en las mujeres de Navarra, mientras que algunos países han comenzado a presentar una tendencia descendente de sus tasas, por ejemplo los Estados Unidos.

Las actividades anti-tabaco deberán se mantenidas e incrementadas si queremos cambiar la tendencia y situarnos en el patrón descendente en el que se encuentran numerosos países.

\section{Cáncer de mama}

En el periodo $2000-2004$, el 3,1\% de las muertes totales y el $14 \%$ de las muertes por cáncer se debieron a esta causa entre las mujeres de Navarra, representando además la tercera causa de muerte prematura.

La mortalidad por cáncer de mama en el periodo 1995 a 2004 muestra una evolución buena en todos los indicadores. A pesar del envejecimiento y del aumento de población, el número de muertes por cáncer de mama ha pasado de 81 al año en el quinquenio 1995-1999 a 74 en el periodo 2000-2004. La tasa ajustada de mortalidad y la tasa ajustada de APVP han disminuido en los mismos periodos: un $17,7 \%$ y $45,4 \%$, respectivamente, indicando que ha disminuido sobre todo la mortalidad entre las mujeres jóvenes (Tablas 3 y 5). Esta tendencia se asemeja a la observada en España y en muchos países de la UE aunque hay que señalar que los descensos de Navarra han sido muy pronunciados.

\section{Cánceres ginecológicos}

Las muertes por cáncer de ovario representan aproximadamente el $1 \%$ de las 
defunciones entre las mujeres y el mismo porcentaje representan los tumores de útero. Los últimos datos parecen señalar una estabilización en las tasas de mortalidad por estos cánceres. Destaca la baja mortalidad por cáncer de cérvix de Navarra, que se corresponde igualmente con bajas tasas de incidencia que han sido documentadas por el Registro de Cáncer de Navarra ${ }^{13}$.

\section{Cánceres digestivos}

El cáncer de estómago continúa mostrando un descenso marcado de sus tasas de mortalidad total y tasa de APVP e incluso del número anual de muertes, y ello a pesar del aumento de población y de su progresivo envejecimiento. Se ha pasado de 105 muertes a 90 muertes al año en los periodos 1995-1999 y 2000-2004. Las tasas ajustadas han descendido un $24 \%$ en ambos sexos y las tasas de APVP todavía más, un $50 \%$ en los hombres y un $36 \%$ en las mujeres. Las tasas de mortalidad por cáncer de estómago de Navarra y España son muy similares y se encuentran por encima de la media de la UE-15 (Tabla 6).

La tasa de mortalidad por cáncer colorrectal es la tercera más alta tras el cáncer de pulmón y próstata en los hombres y la segunda más alta tras el cáncer de mama en las mujeres. La mortalidad por cáncer colorrectal fue más alta en los hombres que en las mujeres en el último quinquenio, 44,5 y 26,4 muertes por 100.000 y además los últimos datos parecen señalar una estabilización. Las tasas de Navarra son muy similares a las de España para este cáncer y se encuentran por debajo de la media de la UE-15 (Tabla 6). Cuando se observan los datos de los Estados Unidos ${ }^{10}$ o los datos globales de la Unión Europea ${ }^{6-10}$ las estadísticas de mortalidad muestran que el cáncer colorrectal está descendiendo. En la Unión Europea entre 1988 y 1996, la mortalidad disminuyó un $12,4 \%$; en los Estados Unidos los datos de mortalidad muestran descensos que se iniciaron en la década de los 70 .

\section{Cáncer de próstata}

La mortalidad por cáncer de próstata, que había presentado una tendencia cre- ciente en la década de los 80 , con una estabilización en los 90, muestra un descenso del $20 \%$ en el último quinquenio. También los datos de España muestran un ligero descenso de las tasas de mortalidad en los últimos años, pasando de 23,7 en al año 1999 a 22,4 muertes por 100.000 en el año $2000^{14-15}$.

En la Unión Europea, las tasas ajustadas de mortalidad por cáncer de próstata más alta se observaron en Suecia y Noruega y las más bajas en Grecia. Las estadísticas de mortalidad de la Unión Europea muestran una estabilización de las tasas en el periodo 1988-1996, mientras que entre la población blanca de los Estados Unidos la mortalidad por cáncer de próstata esta disminuyendo desde principios de la década de los $90^{10}$.

\section{ENFERMEDADES RESPIRATORIAS}

La mortalidad por enfermedades respiratorias de Navarra es similar a la media estatal y de la Unión Europea. La utilización en España desde el año 1999 de la 10 Clasificación Internacional de Enfermedades (CIE-10) ha producido algunos cambios que concretamente afectan a la comparabilidad de las estadísticas de mortalidad por enfermedades respiratorias. Por un lado el traspaso de la insuficiencia respiratoria del capítulo de mal definidas al capítulo de enfermedades respiratorias, aportación que se neutraliza en parte por la pérdida de defunciones en la rúbrica de neumonía por los cambios en las normas de selección de la causa básica de defunción ${ }^{16}$. Los datos de la tabla 2 muestran que las tasas de mortalidad por enfermedades respiratorias de Navarra son más bajas que las de España en ambos sexos.

\section{ENFERMEDADES DIGESTIVAS}

Las tasas de mortalidad por enfermedades del aparato digestivo de Navarra son más bajas que las observadas en España en ambos sexos (Tabla 2). Siguiendo la tendencia observada en la década de los 90 , la mortalidad por úlceras en ambos sexos y la mortalidad por cirrosis han continuado descendiendo. Patrones similares se han observado para estos dos grupos 
de causas de defunción en otras comunidades autónomas y en España ${ }^{14-15}$.

\section{CAUSAS EXTERNAS}

La mortalidad por causas externas entre los hombres de Navarra es 4 veces más alta que entre las mujeres. Son una importante causa de muerte prematura, representando el 30\% de los APVP en los hombres y el $19 \%$ en las mujeres.

Las defunciones por causas externas de mayor relevancia en los hombres de Navarra en el periodo 2000-2004 fueron los accidentes de tráfico, con 67 muertes al año y en segundo lugar los suicidios con 37 muertes al año (Tabla 4). Estas dos causas son además la primera y cuarta causa de años potenciales de vida perdidos, es decir, son causas importantes de muertes prematuras. En el último quinquenio, la mortalidad por accidentes de tráfico entre los hombres ha disminuido un $15 \%$ y la prematura un $32 \%$; mientras que la mortalidad por suicidios se mantiene. A nivel de la UE-15, tanto Navarra como España presentan una tasa de mortalidad por accidentes de tráfico por encima de la media y 3 veces más alta que la observada en el Reino Unido que es el país que tiene mortalidad más baja (Tabla 6). La mortalidad por suicidios de los hombres de Navarra es muy parecida a la de España y las tasas están por debajo de la media de la UE ${ }^{2-3}$.

La tasa de mortalidad por causas externas de las mujeres de Navarra es 3,3 veces más baja que la de los hombres, pero como dato negativo han aumentado un $21 \%$ en el último quinquenio, también la tasa de APVP. Al contrario de lo que ocurre con prácticamente el resto de causas de muerte, la mortalidad por causas externas de las mujeres de Navarra se encuentra por encima de la media de España. Como ocurre entre los hombres Navarra destaca a nivel europeo por su baja mortalidad por suicidios y alta mortalidad por accidentes de tráfico.

\section{ENFERMEDADES INFECCIOSAS}

Las muertes por enfermedades infecciosas representan el 1,7\% de las muertes en los hombres y el $2 \%$ mujeres de Navarra en el periodo 2000-2004. Destaca el des- censo de las tasas de mortalidad por sida, que además afectan fundamentalmente a los jóvenes.

\section{CONCLUSIONES}

\section{Indicadores que presentan una evolución positiva en el periodo 2000-2004}

- En el quinquenio 2000-2004 la esperanza de vida al nacer fue de 77,9 años en los hombres y 85 años en las mujeres, esta última una de las más altas a nivel mundial.

- Han diminuido las tasas de mortalidad en ambos sexos en prácticamente todos los grupos de edad excepto en las mujeres de 15-24 años.

- Ha habido un importante descenso de la mortalidad infantil que se sitúa en 3,3 muertes por 1000 recién nacidos vivos en el periodo 2000-2004 frente a 4,6 en el quinquenio anterior.

- La utilización de tratamientos antirretrovirales más eficaces ha hecho descender las muertes por sida un $60 \%$ en el quinquenio 2000-2004 respecto al anterior.

- Se han conseguido bajas tasas de mortalidad por enfermedades circulatorias en comparación a la mortalidad registrada en España y en la mayoría de los países de la Unión Europea. Además durante 2000-2004 ha continuado descendiendo la mortalidad por isquemia cardiaca, enfermedad cerebrovascular e insuficiencia cardiaca.

- Ha habido un descenso importante de la mortalidad por cáncer de estómago en ambos sexos y estabilización del cáncer colorrectal en los hombres.

- La mortalidad total y mortalidad prematura por cáncer de mama que hasta mediados de los 90 presentaba una tendencia ascendente muestra un descenso de la mortalidad total pero sobre todo de la mortalidad prematura.

- Ha continuado en el periodo 2000-2004 el descenso de la mortalidad por cirrosis y úlceras digestivas en Navarra. 
- Se ha producido un descenso de la mortalidad prematura por accidentes de tráfico entre los hombres.

\section{Indicadores que presentan una evolución negativa en el periodo 2000-2004}

- Fuerte incremento de la mortalidad por cáncer de pulmón entre las mujeres (19\% la mortalidad total y $45 \%$ la mortalidad prematura) que señala el comienzo de la epidemia de cáncer de pulmón entre las mujeres de Navarra.

- Incremento de mortalidad por causas externas entre las mujeres.

\section{BIBLIOGRAFÍA}

1. Instituto de Estadística de Navarra. Población de Navarra calculada a 1 de Julio por grupos quinquenales de edad. Disponible en; http://www.cfnavarra.es/estadistica/agregados/poblacion.

2. Demografia y población. Disponible en: http://www.ine.es/inebase/menu2_dem.htm.

3. Causes of death - Standardised death rate (per 100,000 inhabitants) (Annual Data). Disponible en: http://epp.eurostat.ec.europa.eu.

4. Life expectancy and mortality. Disponible en: http://epp.eurostat.ec.europa.eu.

5. Eurostat. Health Statistics - Key Data on Health 2002. Data 1970-2001. European Communities, 2003.

6. Levi F, Lucchini F, LA Vechia C, Negri E. Trends in mortality from cancer in the European Union, 1995-1994. Lancet 1999; 354: 742-743.

7. LeVi F, Lucchini F, La Vechia C, Negri E. The decline in cancer mortality in the European Union, 1988-1996. Eur J Cancer 2000; 36: 1965-1968.
8. Ferlay J, Bray F, SANKILA R, PARKIn DM. EUCAN: Cáncer Incidence, Mortality and Prevalence in the European Union 1996, version 3.1.IARC CáncerBase No. 4. Lyon, IARCPress, 1999. Limited version available from: URL.

9. TyCZynski JE, BRAY F, AAREleid T, DAlmas M, KuRTinaitis J, Plesko I et al. Lung cancer mortality patterns in selected Central, Eastern and Southern European countries. Int J Cancer 2004; 109: 598-610.

10. Ries LA, Wingo P, Miller D, Howe H, Weir H, RosEnBERG $\mathrm{H}$ et al. The annual report to the nation on the status of cancer, 1973-1997, with a special section on colorectal. Cancer 2000; 88: 2398-2424.

11. BorRas JM, Fernandez E, GonZalez JR, NEGRI E, LUCCHINI F, LA VECCHIA C et al. Lung cancer mortality in European regions (1955-1997). Ann Oncol 2003; 4: 159-161.

12. Fernández E, GonzÁlez JR, BorRas JM, Moreno V, SÁnchez V, PERIS M. Recent decline in cancer mortality in Catalonia (Spain). A joinpoint regression analysis. Eur J Cancer 2001; 37: 2222-2228.

13. Registro de Cáncer de Navarra. Incidencia y mortalidad por cáncer en Navarra, 19931997. Tendencias en los últimos 25 años. An Sist Sanit Navar 2001; 24: 339-362.

14. LLÁCER V, FERnÁNDEZ-CuEnCA R. Mortalidad en España en 1999 y 2000 (I). Boletín Epidemiológico. Centro Nacional de Epidemiología 2003; 11: 109-120.

15. LlÁcer V, FernándeZ-Cuenca R. Mortalidad en España en 1999 y 2000 (II). Boletín Epidemiológico. Centro

16. Ruiz M, Cirera Suárez L, Pérez G, Borrell C, Audica C, Moreno C et al. Comparabilidad entre la novena y la décima revisión de la Clasificación Internacional de Enfermedades aplicada a la codificación de la causa de muerte en España. Gac Sanit 2002; 16: 526532 . 